\title{
Low-frequency radio emission in the massive galaxy cluster MACS J0717.5 + 3745
}

\author{
M. Pandey-Pommier ${ }^{1,2,3,4}$, J. Richard ${ }^{1,2,3,4}$, F. Combes ${ }^{5}$, K. S. Dwarakanath ${ }^{6}$, B. Guiderdoni ${ }^{1,2,3,4}$, C. Ferrari ${ }^{7}$, \\ S. Sirothia ${ }^{8}$, and D. Narasimha ${ }^{9, \star}$ \\ 1 Université de Lyon, 69003 Lyon, France \\ e-mail: mamta.pommier@univ-lyon1.fr \\ 2 Université Lyon 1, Observatoire de Lyon, 9 avenue Charles André, 69230 Saint-Genis Laval, France \\ 3 CNRS, UMR 5574, Centre de Recherche Astrophysique de Lyon, 69007 Lyon, France \\ 4 École Normale Supérieure de Lyon, 69007 Lyon, France \\ 5 LERMA, Observatoire de Paris, 61 avenue de l'Observatoire, 75014 Paris, France \\ 6 Raman Research Institute, 560080 Bangalore, India \\ ${ }^{7}$ Laboratoire Lagrange, UMR7293, Université de Nice Sophia-Antipolis, CNRS, Observatoire de la Côte d'Azur, 06300 Nice, France \\ 8 National Centre for Radio Astrophysics, TIFR, Pune University Campus, Post Bag 3, 411007 Pune, India \\ 9 Theoretical Astrophysics Group, Tata Institute of Fundamental Research, Homi Bhabha Road, 400005 Bombay, India
}

Received 1 May 2013 / Accepted 18 June 2013

\section{ABSTRACT}

\begin{abstract}
Aims. To investigate the nonthermal emission mechanism and their interaction during cluster mergers, we analyze multiple lowfrequency radio data for the X-ray luminous massive galaxy cluster MACS $J 0717.5+3745$, located at $z=0.5548$. Large-scale structure-formation models in the Universe suggest that galaxy clusters grow via constant accretion of gas and the merger of galaxy groups and smaller clusters. Low-frequency radio observations trace these mergers in the form of relics and halos. The dual frequency observations were performed on MACS J0717.5 + 3745 to investigate the spectral index pattern of the nonthermal emission and its interaction within the intracluster medium (ICM), during merger process.

Methods. Continuum observations were carried out using GMRT at 0.235 and $0.61 \mathrm{GHz}$ on MACS J0717.5 + 3745 and archival data from the VLA $(0.074$ and $1.42 \mathrm{GHz})$ and WSRT $(0.325 \mathrm{GHz})$ was used to complement the results. Furthermore, to explore the thermal and nonthermal interactions within the ICM and the morphological distribution, Chandra X-ray and HST data were used. Results. A highly complex nonthermal radio emission distribution is seen in the cluster at very low frequencies, with a global spectral index $\alpha_{0.61}^{0.235} \sim-1.17 \pm 0.37$. We have detected a giant radio halo within the cluster system with a linear size of $1.58 \mathrm{Mpc}$ and a "Chair-shaped" filament structure between the merging subclusters of linear size $853 \mathrm{kpc}$ at $0.235 \mathrm{GHz}$. This is the most powerful halo ever observed with $P_{1.4}=9.88 \times 10^{25} \mathrm{WHz}^{-1}$ and an equipartition magnetic field estimate of $\sim 6.49 \mu \mathrm{G}$. The bright filament structure is well located in the central merging region of subclusters with enhanced temperature, as shown by Chandra and HST data analysis, further indicating the formation of this structure due to shock waves encountered within the ICM during the merger events.
\end{abstract}

Key words. galaxies: clusters: intracluster medium - instrumentation: interferometers - surveys

\section{Introduction}

Cluster of galaxies are the largest gravitationally bound systems in the Universe. They are formed by mergers of smaller clusters and galaxy groups, as well as through continuous accretion of gas. Within the cluster, dark matter represents $\sim 80 \%$ of the gravitating matter followed by $\sim 15 \%$ of diffuse hot gas, and $\sim 5 \%$ is in luminous matter within the galaxies and the embedding intracluster medium (ICM; Feretti et al. 2012). Important progress in the study of ICM and their interactions in galaxy clusters have been made with multi-wavelength observations (Boselli \& Gavazzi 2006; Markevitch \& Vikhlinin 2007); however, less is known about the physical properties and the origin of nonthermal diffuse intracluster components, which also play a vital role in the evolution of galaxy clusters (Dursi \& Pfrommer 2008; Parrish et al. 2009) and which constrain the large-scale structure formation scenario.

In fact, deep radio observations have shown that significant diffuse synchrotron emission is present in some clusters, which indicate there are magnetic fields and cosmic rays within the

\footnotetext{
* Present address: CRAL-l'Observatoire de Lyon, France.
}

cluster volume (Ferretti et al. 2005a; Ferrari et al. 2008; Kale $\&$ Dwarakanath 2009). These diffuse radio sources on different size scales (100 kpc to $>\mathrm{Mpc}$ ) with surface brightness down to $0.1 \mu \mathrm{Jy} \operatorname{arcsec}^{-2}$ at $1.4 \mathrm{GHz}$ are known in about 80 clusters to date at different cluster locations (center, intermediate, periphery) (Feretti et al. 2012; van Weeren et al. 2010). They are further classified as relics, mini-halos, and halos. They are steepspectrum $\left(S(v) \propto v^{-\alpha}, \alpha>1\right)$ sources and represent the best evidence of large-scale magnetic fields and relativistic particles at different cluster locations (relics at the periphery, Giovannini \& Feretti 2004; Bonafede et al. 2009a, mini-halos in the central regions of relaxed clusters; Govoni et al. 2009; Murgia et al. 2010, and halos in non relaxed clusters, Feretti \& Giovannini 2008).

Observational and theoretical evidence favor the hypothesis that the origin of relativistic particles in clusters is related to merging events (so-called electron primary model acceleration). There are several observational probes that agree with primary model predictions. In particular, Mpc scale radio halos have been detected only in merging clusters, showing a correlation between radio and X-ray luminosity and, in a few cases, a spectral steepening as a function of frequency and distance from the 
cluster center (e.g. Giovannini et al. 2009; Venturi et al. 2007, 2008; Brunetti et al. 2008). The radio halo emission is centrally situated and typically unpolarized (except A2255, Govoni et al. 2005 and MACS J0717.5 + 3745, Bonafede et al. 2009b). Furthermore, radio halos are expected to result from electron acceleration due to intracluster turbulence (Brunetti et al. 2001; Petrosian 2001). On the other hand, radio relics are bright elongated or round structures (Feretti et al. 2012) situated at the peripheral regions with variable sizes up to megaparsecs scale. Electrons in radio relics are expected to be accelerated through diffusive shock acceleration (DSA), related to shock waves resulting from merging or accretion shocks in clusters (e.g. Ferrari et al. 2008). Relics are highly polarized ( 20-30\%) and irregular in nature with mostly a steep spectrum $(\alpha \geq 1)$ (Drury 1983; Ensslin et al. 1998; Finoguenov et al. 2010; Miniati et al. 2001; Bruggen et al. 2011). A magnetic field of $\mu \mathrm{G}$ level has been detected in radio relics with relativistic particles in cluster outskirts. Currently 42 clusters show the presence of a radio halo, and 39 clusters host one or a pair or relics (see Feretti et al. 2012, for the most recent compilation of known diffuse cluster radio sources). A few of these clusters (Abell 1240, Abell 2345, etc.) show double relic sources confirming their total detection up to 50 (Feretti et al. 2012; Bagchi 2003).

MACS $J 0717.5+3745$ is a massive X-ray luminous merging cluster, $L_{X}=(24.6 \pm 0.3) \times 10^{44} \mathrm{erg} \mathrm{s}^{-1}$ with ICM temperature $11.6 \pm 0.5 \mathrm{keV}$ located at $z=0.5548$, discovered in the Massive Cluster Survey (MACS; Ebeling et al. 2001; Edge et al. 2003; Ebeling et al. 2007). It has a very complex morphological distribution showing multiple mergers with shock regions, known at high redshift so it serves as a most promising target for studies of physical mechanisms driving merger events. Ebeling et al. (2004) discovered a $6 \mathrm{Mpc}$ filamentary substructure leading into the cluster from the southeast with optical observations from Subaru $8 \mathrm{~m}$ telescope. Ma et al. (2008, 2009) present optical (Hubble Space Telescope (HST), ACS; Keck-II, DEIMOS) and $\mathrm{X}$-ray (Chandra) observations, and identified four distinct components in MACS J0717.5 + 3745 undergoing a triple merger event, instead of the typically seen two cluster mergers, e.g., $1 E S 0657$ - 56 (Markevitch et al. 2002). Ma et al. analyzed the galaxy and gas distribution with radial velocity information and found that the merger events may have formed the large-scale filament to the southeast of the cluster. Further, the ongoing merger give rise to shock heating, which in turn increases the ICM temperature as measured in X-ray (Chandra) observations. The contiguous accretion of gas along the cluster-filament interface may give rise to the large size $(\sim 1 \mathrm{Mpc})$ of the high-temperature ICM region, as measured in MACS J0717.5 + 3745 X-ray observations. The cluster is known to have disturbed morphological distribution with the largest Einstein radius, $\theta_{\mathrm{e}} \sim 55^{\prime \prime}$ and shallow surface mass distribution (Zitrin et al. 2009). In strong lensing analysis studies four mass peaks were located, in agreement with optically identified regions by (Ma et al. 2008, 2009; Limousin et al. 2012). The Sunyaev-Zel'dovich effect (SZE) studies of the cluster were performed by Mroczkowski et al. (2012) with the MUSTANG bolometer array on the Green Bank Telescope (GBT) between 90-268 GHz. The MUSTANG SZE data confirm that a pressure enhancement is seen in the shockheated gas $(\geq 20 \mathrm{keV})$ immediately adjacent to the extended radio emission as seen in low-frequency radio maps of this cluster and as previously indicated from Chandra observations.

The radio observations of the cluster have been equally interesting with the discovery of a relic structure by (Edge et al. 2003), from the Faint Images of the Radio Sky (FIRST) at $20 \mathrm{~cm}$ (Becker et al. 1995) with no radio halo structure found in the data owing to higher noise. Bonafede et al. 2009b conducted multi-frequency and multi-channel Very Large Array (VLA) observations at frequencies spanning $1.365-4.885 \mathrm{GHz}$ to further probe the cluster morphology and discovered a giant radio halo of $1 \mathrm{Mpc}$ at $4.885 \mathrm{GHz}$ and $1.5 \mathrm{Mpc}$ at $1.365 \mathrm{GHz}$ along with the $20 \%$ polarized radio substructure. The lack of Faraday rotation in the observations is not consistent with the assumption of relic produced by a merger shock near the center of the cluster. Likewise, no steepening of the spectral index across the short axis of the substructure is seen, as would be expected of a radio relic following a merger. Bonafede et al. thus argue that the substructure is not a radio relic, but rather a bright, polarized filament connected with the radio halo.

A global spectral index for the entire diffuse radio emission of $\alpha_{4.9}^{1.42 \mathrm{GHz}}=-1.27 \pm 0.02$ with an equipartition magnetic field estimate of $\sim 1.2 \mu \mathrm{G}$ was derived by assuming JaffePerola emission model for the integrated radio spectra of the cluster (Jaffe \& Perola 1973). Van Weeren et al. (2009) conducted GMRT observations at $0.614 \mathrm{GHz}$ to study the diffuse radio emission within the cluster and found a radio relic of $700 \mathrm{kpc}$ and giant radio halo of $1.2 \mathrm{Mpc}$ with a global spectral index of, $\alpha_{4.9}^{0.61 \mathrm{GHz}}=-1.24 \pm 0.05$. The equipartition magnetic field of $5.8 \mu \mathrm{G}$ was derived at the location of the radio halo from these observations. According to van Weeren et al. (2009), during the merger events, the electrons within the cluster that are accelerated by diffuse shock acceleration mechanism have formed the bright central radio substructure. However, the spectral index over the bright substructure varies roughly between -0.8 and -1.5 , so it does not fit with the merger shock scenario. In fact, the Mach number values calculated using the spectral index information lies between 1.7 to 2.8 , indicating that the relic might instead trace an accretion shock. Furthermore, the hightemperature regions of the ICM are cospatial with the bright central radio substructure that is perpendicular to the merger axes of the cluster and large-scale filament, which does not rule out the possibility that the relic might trace the merger shock scenario either. However such merger shocks are seen in clusters typically with radii less than $1 \mathrm{Mpc}$, unlike to MACS J0717.5+3745 where a cluster radii greater than $1 \mathrm{Mpc}$ is measured (Bonafede et al. 2009a,b; van Weeren et al. 2009). Feretti et al. (2012) have carried out a statistical study of diffuse radio emission in galaxy clusters and do not classify the bright central radio substructure as a "relic" due to its location in the cluster central region.

The main interest of our study for radio emission arising from galaxy clusters and ICM is to explore their spectral nature at very low frequencies (down to $0.235 \mathrm{GHz}$ ). With the highsensitivity data at $0.235 \mathrm{GHz}$ we aim to get better estimates of luminosity and magnetic field, one of the fundamental ingredients in the physics of the ICM. By combining multi-frequency radio, Chandra, and HST archival data, it is possible to derive important correlations between shock regions and nonthermal emissions. Ferreti et al. (2012) and Murgia et al. (2010), studied the link between the cluster X-ray properties, their evolutionary stage, and their nonthermal emission. These studies have been crucial for a comprehensive physical description of the intracluster medium, and they suggest that nonthermal emission plays an important role in the cluster formation and evolution.

In this paper we present 0.235 and $0.61 \mathrm{GHz}$ continuum observations of MACS J0717.5 + 3745 with the Giant Metrewave Radio Telescope (GMRT). These observations are complemented by VLA and Westerbork Synthesis Radio Telescope (WSRT) archival observations. In Sect. 2 we give a brief overview of radio observations and data reduction. In Sect. 3, the radio images, spectral index maps, and spectra of discrete field 
Table 1. GMRT observations and data from archive.

\begin{tabular}{lcccccc}
\hline \hline Observation & $V L S S^{a}$ & GMRT $^{b}$ & WSRT & GMRT $^{c}$ & NVSS $^{d}$ & FIRST $^{e}$ \\
\hline Frequency (MHz) & 74 & 235 & 325 & 610 & 1420 & 1420 \\
Bandwidth (MHz) & 1.56 & $2 \times 16$ & 120 & $2 \times 16$ & $2 \times 50$ & 50 \\
Channel width & $122 \mathrm{KHz}$ & $125 \mathrm{kHz}$ & $5 \mathrm{MHz}$ & $125 \mathrm{kHz}$ & $50 \mathrm{MHz}$ & $3 \mathrm{MHz}$ \\
Observation dates & Archive & $5 \mathrm{Nov.}, 2012$ & Archive & $5 \mathrm{Nov} ., 2012$ & Archive & Archive \\
Total on-source time (h) & - & $\sim 5$ & - & $\sim 5$ & - & - \\
Synthesized beam $(\mathrm{arcsec})$ & $80 \times 80$ & $13 \times 11$ & $54 \times 54 \operatorname{cosec} \delta$ & $5.7 \times 4.8$ & $45 \times 45$ & $5 \times 5$ \\
rms noise, $\sigma\left(\mathrm{mJy} \mathrm{beam}^{-1}\right)$ & 73 & 0.65 & 3.1 & 0.048 & 0.42 & 15 \\
\hline
\end{tabular}

References. ${ }^{(a)}$ Cohen et al. (2007), ${ }^{(b)}$ 23rd cycle observations, ${ }^{(c)}$ Rengelink et al. (1997), ${ }^{(d)}$ Condon et al. (1998), ${ }^{(e)}$ Becker et al. (1995).

sources are presented. Finally the discussion and conclusions are presented in Sects. 4 and 5. Throughout this paper we assume a $\Lambda \mathrm{CDM}$ cosmology with $H_{0}=71 \mathrm{~km} \mathrm{~s}^{-1} \mathrm{Mpc}^{-1}, \Omega_{\mathrm{M}}=0.3$, and $\Omega_{\Lambda}=0.7$. At the distance of MACS J0717.5 + 3745 $(z=0.5548), 1^{\prime \prime}$ corresponds to $6.348 \mathrm{kpc}^{1}$.

\section{Observation and data reduction}

We have observed the massive cluster MACS J0717.5 + 3745 in the 23rd observation cycle using the GMRT at 0.235 , and $0.61 \mathrm{GHz}$. The antennas were pointed at RA $=07^{\mathrm{h}} 17^{\mathrm{m}} 32.63^{\mathrm{s}}$ and Dec $=+37^{\circ} 44^{\prime} 59.70^{\prime \prime}$ (J2000). The visibilities were acquired at 128 spectral channels in continuum mode, in order to have higher signal-to-noise ratio $(\mathrm{S} / \mathrm{N})$ and thus reduce the narrow band radio frequency interferences (RFIs). The details of the observations are summarized in Table 1, where we provide the frequency, total bandwidth, observation date, total time on source, beamwidth (FWHM) of the full array, and rms level $(1 \sigma)$ in the full resolution images. The visibility data was processed (calibration, flagging, imaging) with the NRAO's Astronomical Image Processing System (AIPS) software.

\subsection{GMRT observations at 0.235 and $0.614 \mathrm{GHz}$}

The 0.235 and $0.614 \mathrm{GHz}$ observations consist of one data set each of 5.0h on source, collected on 5 November 2012. The observations were performed for both the lower side band (LSB) and the upper side band (USB) with a total bandwidth of $32 \mathrm{MHz}$ split into 128 channels at $0.61 \mathrm{GHz}$ and $8 \mathrm{MHz}$ splitted into 60 channels at $0.235 \mathrm{GHz}$. The flux density scale was calibrated by the primary calibrators 3C 147 and 3C 286. The source $0735+331$ was observed for 6 -min scan at intervals of $\sim 50 \mathrm{~min}$ and used as the secondary phase and gain calibrator. The bandpass was calibrated using the sources 3C 286 and 3C 147 . About $26 \%$ of the dataset that was affected by RFIs was flagged using task FLGIT. Both data sets were averaged in frequency to eight channels to reduce bandwidth smearing effect and facilitate RFI flagging. Several cycles of imaging and phase self-calibration was applied to the data to produce initial phase corrected images. A final amplitude and phase selfcalibration run was performed to obtain the final amplitude and phase-corrected images. At $0.61 \mathrm{GHz}$, both the IFs were simultaneously gridded, flagged, cleaned, and imaged, while only one IF was available for imaging at $0.235 \mathrm{GHz}$. To minimize the effects of non-coplanar baselines while imaging in AIPS, the polyhedron method (Perley 1989; Cornwell \& Perley 1992) was used. To remove the sidelobes effect from the bright sources away from the field center, the image size of twice the primary

\footnotetext{
1 http://www . astro.ucla.edu/ wright/CosmoCalc.html
}

beam radius was cleaned using the $3 \mathrm{D}$-technique with multiple facets to account for the non-coplanarity of the incoming wavefront within the large primary beam.

Natural weighting was applied to each dataset using a robust parameter of 0.5 (Briggs 1995), to give constant weights to all visibilities and pick up the diffuse structure. The contour levels of the full-resolution 0.235 and $0.614 \mathrm{GHz}$ image are shown in the Fig. 1. The radio image at $0.614 \mathrm{GHz}$ has FWHM beam of $5.70^{\prime \prime} \times 4.82^{\prime \prime}$ and a noise level of $0.048 \mathrm{mJy} /$ beam $(1 \sigma)$, and at $0.235 \mathrm{GHz}$ has FWHM beam of $13.46^{\prime \prime} \times 10.76^{\prime \prime}$ and a noise level of $0.65 \mathrm{mJy} /$ beam $(1 \sigma)$. Figure 1 also shows the high-resolution VLA map at $1.4 \mathrm{GHz}$ obtained from the FIRST survey archive (Becker et al. 1994), with resolution similar to $0.61 \mathrm{GHz}$ map. We have labeled the brightest radio sources close to the diffuse emissions alphabetically in order to further clarify the discussion on the association of these sources with the halo (see Fig. 1).

The theoretical noise in the image is given by

$$
\sigma_{\text {thermal }}=\frac{\sqrt{2} T_{\text {sys }}}{G \sqrt{n(n-1) N_{\mathrm{IF}} \Delta v t_{\mathrm{int}}}}
$$

where $T_{\text {sys }}$ is the system temperature for GMRT $=92 \mathrm{~K}$ at $0.61 \mathrm{GHz}$ and $177 \mathrm{~K}$ at $0.235 \mathrm{GHz}, N_{\mathrm{IF}}$ is the number of side bands recording both $\mathrm{RR}$ and $\mathrm{LL}$ polarizations $=1$ at $0.61 \mathrm{GHz}$ and 0.5 at $0.235 \mathrm{GHz}, \Delta v$ the bandwidth per sideband $=13.5 \mathrm{MHz}$ at $0.61 \mathrm{GHz}$ and $6.9 \mathrm{MHz}$ at $0.235 \mathrm{GHz}, n$ the number of working antennas $=27$ at both the frequencies, $G$ the antenna gain $=0.32 \mathrm{~K} \mathrm{Jy}^{-1}$ at $0.61 \mathrm{GHz}$ and $0.33 \mathrm{~K} \mathrm{Jy}^{-1}$ at $0.235 \mathrm{GHz}$ and $t_{\text {int }}$ is the net integration time five hours on the target source. The expected theoretical thermal noise at $0.61 \mathrm{GHz}$ is typically $31 \mu \mathrm{Jy}$, and at $0.235 \mathrm{GHz}$ is typically $115 \mu \mathrm{Jy}$. The noise recorded in our maps are $48 \mu \mathrm{Jy}$ at $0.61 \mathrm{GHz}$ and $650 \mu \mathrm{Jy}$ at $0.235 \mathrm{GHz}$, so an increase in the noise by $\sim 1.5$ and $\sim 5.6$ times is seen at 0.61 and $0.235 \mathrm{GHz}$, respectively. Due to natural weighting applied to the data during imaging, an increase in the recorded noise level by typically $10 \%$ as compared to the theoretical noise is seen. Further residual errors in the noise estimation are most likely to be attributed to RFI and increase in sky temperature (dominating at lower frequencies), sidelobes from bright sources, and residual errors from calibration.

\subsection{Archive data from VLA and WSRT}

To investigate the multi-frequency extended radio emission from MACS J0717.5+3745 we have used the archival low resolution data available from the WENSS survey (Rengelink et al. 1997) at $0.325 \mathrm{GHz}$, the VLA at $0.074 \mathrm{GHz}$ - the VLSS survey (Cohen et al. 2007) and 1.4 GHz VLA's NVSS survey (Condon et al. 1998). The details of the survey parameters are listed in Table 1. 

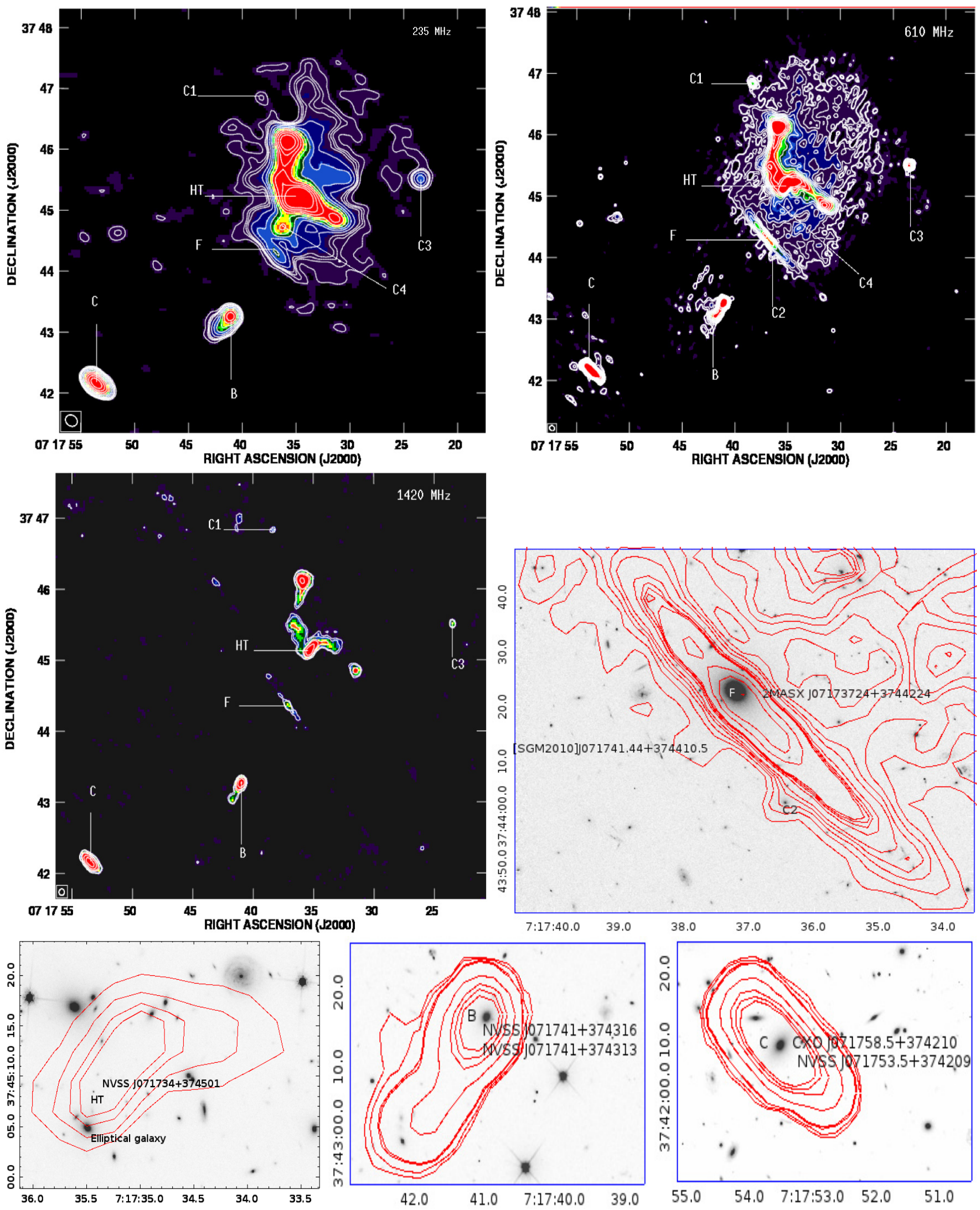

Fig. 1. from top left to middle left -3 panels: high resolution radio images of massive galaxy cluster MASCJ0717.5 $+3745(z=0.5548)$ at $0.235 \mathrm{GHz}$ from GMRT (top left) at FWHM beam of $13.46^{\prime \prime} \times 10.76^{\prime \prime}$ resolution, $0.61 \mathrm{GHz}$ from GMRT (top right) at FWHM of 5.70" $\times 4.82^{\prime \prime}$ resolution and $1.42 \mathrm{GHz}$ from FIRST survey (middle left) at FWHM of $5^{\prime \prime} \times 5^{\prime \prime}$ resolution. Contours start at $(3 \sigma)$ and scale by a factor of $\sqrt{2}$. From middle right to bottom panels: finding charts from HST for source $\mathrm{F}(z=0.1546)$, HT, B and C with overlaid $0.61 \mathrm{GHz}$ radio contours. 

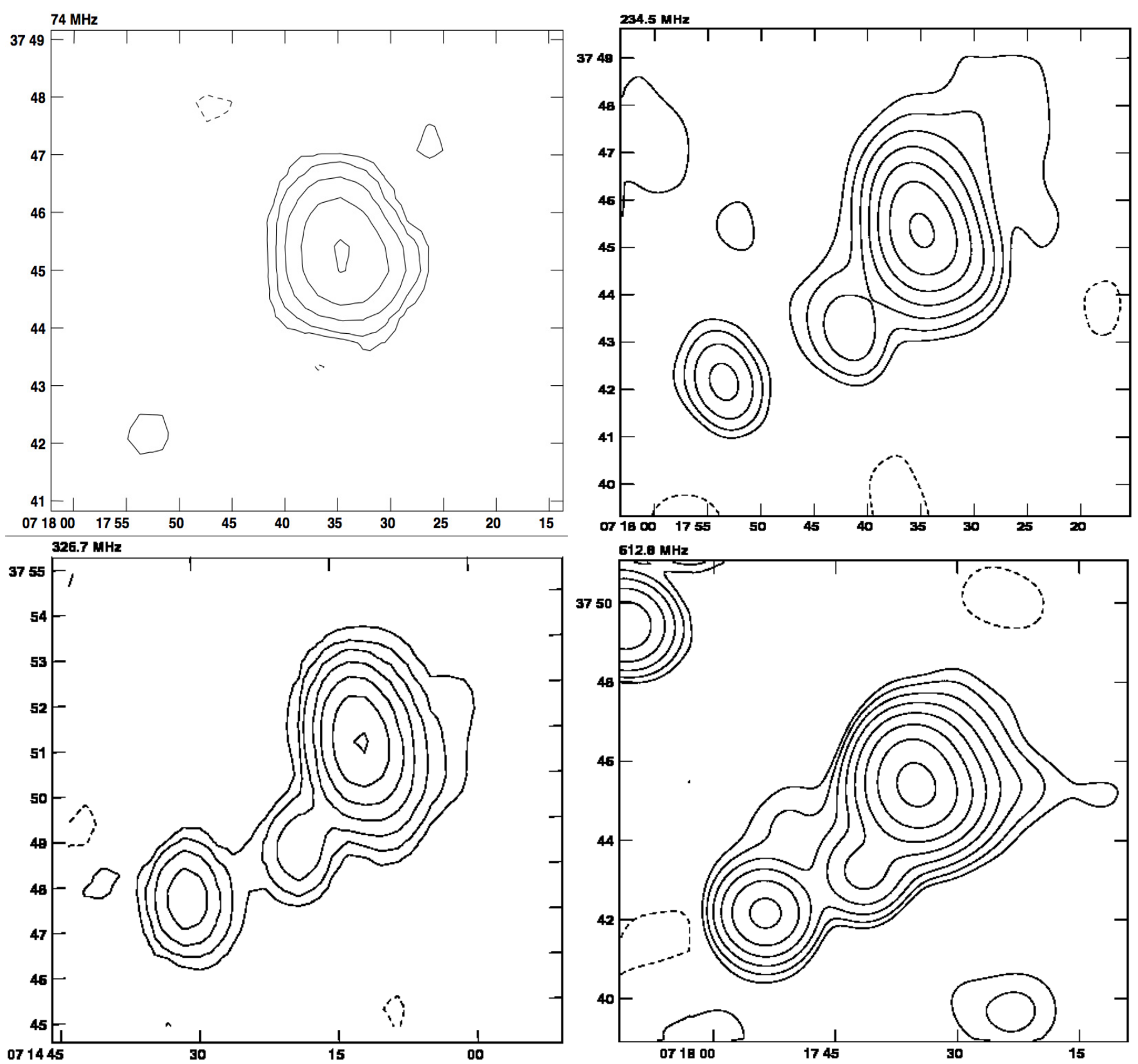

Fig. 2. Low resolution radio images of massive galaxy cluster MACS $J 0717.5+3745$ with contours starting at $(-3 \sigma, 3 \sigma)$ and scaling by a factor of 2 with coordinates in the map at BJ2000 for same sky area. Left to right: $0.074 \mathrm{GHz}$ (VLSS survey; $\sigma=73 \mathrm{mJy} /$ beam; resolution $=80^{\prime \prime} \times 80^{\prime \prime}$ ), $0.234 \mathrm{GHz}$ (GMRT; $\sigma=5 \mathrm{mJy} / \mathrm{beam}$; with resolution similar to WSRT), $0.325 \mathrm{GHz}$ (WSRT/ WNESS survey, $\sigma=3 \mathrm{mJy} / \mathrm{beam}$; resolution $=54^{\prime \prime} \times 84^{\prime \prime}$; Coordinates in the map at BJ1950), $0.612 \mathrm{GHz}$ (GMRT, $\sigma=0.69 \mathrm{mJy} /$ beam; with resolution similar to WSRT).

The degraded resolution maps at lower frequencies increases the sensitivity to fully measure the arcmin size diffuse structure emission that lies fully within the primary beam. Figure 2 shows the diffuse radio emission at various frequencies from MACS $J 0717.5+3745$. The GMRT images at 0.235 and $0.614 \mathrm{GHz}$ are convolved with poorer beam resolution in order to produce a low-resolution map comparable to the $0.325 \mathrm{GHz}$ map.

\section{Results: optical and radio properties, images and spectral analysis}

We analyzed the results of the GMRT observations with particular emphasis on the study of diffuse emission in our high-sensitivity maps at $0.61 \mathrm{GHz}$ and new image at $0.235 \mathrm{GHz}$. These observation will help us determine the total integrated radio spectrum down to $0.235 \mathrm{GHz}$ for the cluster halo, bright central filament structure, and discrete field sources (HT, F, B, $\mathrm{C}, \mathrm{C} 1, \mathrm{C} 2, \mathrm{C} 3$ and $\mathrm{C} 4$, see Fig. 1) that are related to the cluster and the large-scale optical and X-ray filaments. The new $0.235 \mathrm{GHz}$ map allows us to derive much better the spectral index, and therefore the various probable (re-)acceleration mechanisms, as steep spectra index implies aged radio plasma, with no recent re-acceleration activity. Furthermore, the low-frequency image at 0.235 and $0.61 \mathrm{GHz}$ allows us to explore the nature of two new contaminating steep spectrum field sources (C2 and C4) within the halo, which are often not detected at higher frequencies. These compact sources do not contribute diffuse emission 
Table 2. Integrated spectra for the halo and "Chair-shaped" filament structures.

\begin{tabular}{lcccc}
\hline \hline Source & $\begin{array}{c}\text { Beam } \\
" \times^{\prime \prime}\end{array}$ & $\begin{array}{c}v \\
(\mathrm{GHz})\end{array}$ & $\begin{array}{c}\text { Flux density } \\
(\mathrm{mJy})\end{array}$ & $\begin{array}{c}\text { Size } \\
(\mathrm{Mpc})\end{array}$ \\
\hline "Chair-shaped" filament & $18 \times 14$ & 4.8 & $16.00^{*}$ & - \\
& $5 \times 4$ & 1.42 & $83.00^{*}$ & 0.83 \\
& $5.70 \times 4.82$ & 0.61 & $260 \pm 3$ & $\left(1.9^{\prime}=\right) 0.772$ \\
& $84 \times 54$ & 0.325 & - & - \\
$13.46 \times 10.76$ & 0.235 & $844 \pm 8$ & $\left(2.1^{\prime}=\right) 0.853$ \\
& $80 \times 80$ & 0.074 & - & - \\
\hline Halo & $18 \times 14$ & 4.8 & $26 \pm 1^{*}$ & 1 \\
& $21 \times 18$ & 1.42 & $118 \pm 5^{*}$ & 1.5 \\
& $13.34 \times 11.05$ & 0.61 & $162 \pm 0.23$ & $\left(3.7^{\prime}=\right) 1.5$ \\
& $84 \times 54$ & 0.325 & $1200 \pm 0.5^{*}$ & 1.8 \\
& $25.45 \times 22.31$ & 0.235 & $492.5 \pm 0.52$ & $\left(3.9^{\prime}=\right) 1.58$ \\
& $80 \times 80$ & 0.074 & $5800 \pm 0.6$ & 1.3 \\
\hline
\end{tabular}

References. ${ }^{(*)}$ Bonafede et al. (2009b).

from the halo and have different origins, so they were subtracted from the data during the flux density measurements. The flux density of radio halo, bright central filament structure, and field sources (HT, B, and F) at the different frequencies has been determined by using AIPS task IMSTAT, owing to their extended nature. The task JMFIT was used to determine the flux density of unresolved sources (C, C1, C2, C3, and C4). We determined the flux density by integrating their radio brightness down to the $3 \sigma$ level.

\subsection{The "Chair-shaped" filament structure}

The high-resolution images at $0.61 \mathrm{GHz}$ and $0.235 \mathrm{GHz}$ (Fig. 1) reveal a bright elongated substructure or "Chair-shaped" filament that was discovered by Edge et al. 2003 as the "relic" within the cluster. This bright elongated substructure runs from north to south with a twist towards southwest. Enhanced radio emission is measured in the north, center, and south ends of this structure. The linear size of the entire structure is $853 \mathrm{kpc}$ at $0.235 \mathrm{GHz}$ and $772 \mathrm{kpc}$ at $0.61 \mathrm{GHz}$ in GMRT maps. The angular size measured in the map was converted into linear size using constants $H_{0}, \Omega_{M}$, and $(z=0.5548)$ as mentioned in Sect. 1 . We have provided better size estimates (about $\sim 70 \mathrm{kpc}$ more) with our maps than did van Weeren et al. (2009) thanks to higher sensitivity achieved in our observations. The FIRST survey map at 1.4 GHz shows the "Chair-shaped" filament with a total linear size of $731 \mathrm{kpc}$. The physical properties of this bright central filament derived from high-resolution maps are listed in Table 2. In addition to the "Chair-shaped" structure, Fig. 1 also shows the associated field source HT, located midway along the elongated central filament and its detection at optical wavelength. Source HT is identified as a "head-tail" source from radio observations by van Weeren et al. (2009). We confirm having detected the tail structure down to $0.235 \mathrm{GHz}$ and steepening in the spectral index map (Fig. 3), as expected at lower frequencies. We identified radio galaxy NVSS $J 071734+374501$, from the Nasa/IPAC Extra-galactic Database (NED) as a likely counterpart for source HT. No optical counterpart was detected in DSS2-red map by Edge et al. (2003). However, the core of the radio galaxy was identified as coincident with the optical counterpart in the HST image with a spectral index of $\sim 0.7$ (Bonafede et al. 2009b). Van Weeren et al. (2009) went on to identify an elliptical galaxy located within the cluster at RA $07^{\mathrm{h}} 17^{\mathrm{m}} 35^{\mathrm{s}} .5$, Dec $+37^{\circ} 45^{\prime} 0.5^{\prime \prime} .5$ as a likely counterpart. A steep spectral index of -1.55 was derived from low-resolution image by them indicating that the emission from the tail region is partially contaminated by the surrounding emission from the "Chair-shaped" filament region. Likewise, the unresolved source $\mathrm{C} 4$ located midway below this structure is a new detection in our $0.235 \mathrm{GHz}$ that was not detected at $0.61 \mathrm{GHz}$. The source is associated to compact AGN from the NED catalog, and it has a highly steep spectrum with cut-off frequency at $0.61 \mathrm{GHz}$ (see Fig. 3 bottom panel).

\subsection{The extended radio halo}

With the high sensitivity and higher resolution observation performed with the GMRT at 0.61 and $0.235 \mathrm{GHz}$, we have managed to map the most distant halo within the galaxy cluster MACS J0717.5 $3745(z=0.5548)$ down to $0.235 \mathrm{GHz}$. The diffuse extended radio halo emission is detected throughout the bright central radio substructure with angular sizes of $3.7^{\prime}$ and $3.9^{\prime}$ at 0.61 and $0.235 \mathrm{GHz}$, respectively. This corresponds to a total size of about $1.50 \mathrm{Mpc}$ at $0.61 \mathrm{GHz}$ and $1.58 \mathrm{Mpc}$ at $0.235 \mathrm{GHz}$, using $H_{0}, \Omega_{M}$, and $(z=0.5548)$ as mentioned in Sect. 1. Because the noise is high in the FIRST survey data at $1.4 \mathrm{GHz}$ (Fig. 1), the diffuse structure is not detected, though the resolution is similar to GMRT at $0.61 \mathrm{GHz}$. However, the NVSS low-resolution map at $1.4 \mathrm{GHz}$ shows a diffuse halo structure of roughly $\sim 4$ arcmin (corresponding to a linear size of $1.58 \mathrm{Mpc}$ ), while the apparent angular extent of the halo in the WNESS image is $\sim 4.67$ arcmin, corresponding to $\sim 1.8 \mathrm{Mpc}$ (Bonafede et al. 2009 b). Table 2 shows the physical properties of the halo structures at multi-frequency that are derived from lower resolution maps that allows the diffuse structure tobe best extracted.

The low-resolution maps in Fig. 2 show that the extended halo structure is clearly detected at $0.074,0.235,0.325$, and $0.61 \mathrm{GHz}$ with resolution $\left(\sim 54^{\prime \prime} \times 84^{\prime \prime}\right)$ similar to WSRT at $0.325 \mathrm{GHz}$ or lower. The $0.61 \mathrm{GHz}$ map has high sensitivity to the extended emission as compared to the $0.235 \mathrm{GHz}$ map owing to a reduced noise level of about $0.69 \mathrm{mJy} /$ beam. At $0.074 \mathrm{GHz}$, a less diffuse structure is detected around the central halo due to the lower sensitivity of $73 \mathrm{mJy} /$ beam.

\subsection{The discrete field sources}

In addition to the "Chair-shaped" filament, source HT, compact sources $\mathrm{C} 4$, and halo structure, Fig. 1 and Table 3 also show the associated field sources $(\mathrm{C} 1, \mathrm{C} 2, \mathrm{C} 3, \mathrm{~F}, \mathrm{~B}, \mathrm{C})$ and their detection at optical wavelengths. An elongated source $\mathrm{F}$ is detected at the southwest edge of the halo, below the cluster center. This source 
Table 3. Integrated spectra for field sources.

\begin{tabular}{|c|c|c|c|c|c|c|}
\hline Source & $\begin{array}{l}\text { RA } \\
\text { Dec }\end{array}$ & $\begin{array}{c}v \\
(\mathrm{GHz})\end{array}$ & $\begin{array}{c}\text { Flux density } \\
(\mathrm{mJy})\end{array}$ & Morphology & $\begin{array}{l}\text { Counterparts } \\
\text { from NED }\end{array}$ & $z$ \\
\hline Source- HT & $\begin{array}{l}07^{\mathrm{h}} 17^{\mathrm{m}} 32.64^{\mathrm{s}} \\
37^{\circ} 44^{\prime} 59.66^{\prime \prime}\end{array}$ & $\begin{array}{c}4.8 \\
1.42 \\
0.61 \\
0.325 \\
0.235 \\
0.074\end{array}$ & $\begin{array}{c}3.9^{*} \\
22.4^{*} \\
109.8 \pm 3.2 \\
- \\
573 \pm 7 \\
-\end{array}$ & head-tail & $\begin{array}{c}\text { Elliptical galaxy } \\
\text { NVSS } J 071734+374501\end{array}$ & $\begin{array}{l}\text { unknown } \\
\text { unknown }\end{array}$ \\
\hline Source- F & $\begin{array}{l}07^{\mathrm{h}} 17^{\mathrm{m}} 36.86^{\mathrm{s}} \\
37^{\circ} 44^{\prime} 19.66^{\prime \prime}\end{array}$ & $\begin{array}{c}4.8 \\
1.42 \\
0.61 \\
0.325 \\
0.235 \\
0.074 \\
\end{array}$ & $\begin{array}{c}- \\
9.08^{*} \\
30.29 \pm 0.5 \\
10.17^{*} \\
63.17 \pm 3 \\
219.24^{a}\end{array}$ & core+lobes & 2MASX J07173724 + 3744224 & 0.1546 \\
\hline Source- B & $\begin{array}{l}07^{\mathrm{h}} 17^{\mathrm{m}} 41.07^{\mathrm{s}} \\
37^{\circ} 43^{\prime} 14.65^{\prime \prime}\end{array}$ & $\begin{array}{c}4.8 \\
1.42 \\
0.61 \\
0.325 \\
0.235 \\
0.074 \\
\end{array}$ & $\begin{array}{c}6.40^{*} \\
18.3^{*} \\
44.79 \pm 2.6 \\
61.22^{*} \\
100.93 \pm 0.8 \\
219.24^{a} \\
\end{array}$ & head-tail & $\begin{array}{l}\text { NVSS J071741 + } 374316 \\
\text { NVSS J071741 + } 374313\end{array}$ & $\begin{array}{l}\text { unknown } \\
\text { unknown }\end{array}$ \\
\hline Source- C & $\begin{array}{l}07^{\mathrm{h}} 17^{\mathrm{m}} 53.50^{\mathrm{s}} \\
37^{\circ} 42^{\prime} 09.55^{\prime \prime}\end{array}$ & $\begin{array}{c}4.8 \\
1.42 \\
0.61 \\
0.325 \\
0.235 \\
0.074 \\
\end{array}$ & $\begin{array}{c}14.70^{*} \\
32.30^{*} \\
75.96 \pm 1 \\
115.18^{*} \\
109.75 \pm 0.6 \\
219.24^{a} \\
\end{array}$ & unresolved & $\begin{array}{c}\text { CXO J071753.5 + } 374210 \\
\text { NVSS J071753.3 + } 374209\end{array}$ & $\begin{array}{l}\text { unknown } \\
\text { unknown }\end{array}$ \\
\hline Source- C2 & $\begin{array}{l}07^{\mathrm{h}} 17^{\mathrm{m}} 36.37^{\mathrm{s}} \\
37^{\circ} 44^{\prime} 03.37^{\prime \prime}\end{array}$ & $\begin{array}{c}4.8 \\
1.42 \\
0.61 \\
0.325 \\
0.235 \\
0.074 \\
\end{array}$ & $\begin{array}{c}- \\
- \\
0.98 \pm 0.05 \\
- \\
1.95 \pm 0.5^{a} \\
- \\
\end{array}$ & unresolved & & unknown \\
\hline Source- C4 & $\begin{array}{l}07^{\mathrm{h}} 17^{\mathrm{m}} 36.26^{\mathrm{s}} \\
37^{\circ} 44^{\prime} 43.80^{\prime \prime}\end{array}$ & $\begin{array}{c}4.8 \\
1.42 \\
0.61 \\
0.325 \\
0.235 \\
0.074\end{array}$ & $\begin{array}{c}- \\
- \\
0.15 \pm 0.1^{a} \\
- \\
29.64 \pm 0.7 \\
-\end{array}$ & unresolved & & unknown \\
\hline
\end{tabular}

Notes. ${ }^{(a)}=3 \sigma$ upper limit from the map, ${ }^{(*)}$ Bonafede et al. (2009b).

is identified at redshift $z=0.1546$ (van Weeren et al. 2009) as an FR-I type (Fanaroff \& Riley 1974) source with a compact core and diffuse linear extension (on both sides) of $\sim 1^{\prime}$ from GMRT $0.61 \mathrm{GHz}$ data (van Weeren et al. 2009). We have identified a radio galaxy, 2MASX $J 07173724+374422$, a counterpart for source F from NED catalog. It is interesting to note that another radio galaxy, [S GM2010]071741.44 + 374410.5, located at redshift $z=0.55$ is also identified in the radio map. We also confirm the identification of the faint compact source $\mathrm{C} 2$ by van Weeren et al. (2009). This source is buried in the extended emission from source $\mathrm{F}$ and has AGN as counterparts.

Two slightly resolved sources, B and C, are detected outside the halo in the southwest direction along the X-ray and optical large-scale filament (Ebeling et al. 2004). Source B has a compact core with "head-tail" morphology and has two variable radio galaxies (NVSS $J 071741+374316$, NVSS J071741+ 374313) identified as counterparts from the NED catalog. For the unresolved source C, two radio galaxies (CXO J071753.5 + 374210 and NVSS J071753.3 + 374209) are also identified as NED counterparts.

The main properties of the identified radio galaxies along with their position coordinates, flux densities, morphology, and counterparts, are listed in Table 3 . We need to take the possible contamination from these radio galaxies embedded within the diffuse emission into account, in order to conduct a proper study of the diffuse emission associated with the ICM. The compact field sources (C1 and $\mathrm{C} 3$ ) are not listed in the table, since they are not within the area of the halo (source $\mathrm{C} 2$ ad $\mathrm{C} 4$ are merged inside the halo emission) and do not show extended diffuse structures, hence do not contribute to the halo emission.

Furthermore, the low-resolution images (Fig. 2) reveal a bridge of diffuse radio emission, which connects the radio galaxies $\mathrm{B}$ and $\mathrm{C}$ with the cluster halo. This connecting diffuse radio emission bridge has an angular size of $\sim 9$ arcmin, which transforms to a linear scale size of $3.66 \mathrm{Mpc}$ and is coincident with the large-scale filament funneling matter onto MACS J0717.5+ 3745 , which has been detected at optical and X-ray wavelengths by Ebeling et al. (2004) and Ma et al. (2009). This bridge emission is best detected at $5 \sigma$ level in the $0.61 \mathrm{GHz}$ low-resolution map thanks to its high sensitivity, and it confirms the detection at $0.325 \mathrm{GHz}$ by Bonafede et al. (2009b). At $0.234 \mathrm{GHz}$ and $0.074 \mathrm{GHz}$, the bridge emission is partially detected since the noise is comparatively higher in the map. The faint bridge emission is not detected in the higher resolution VLA map at 
$1.4 \mathrm{GHz}$, as compared to $0.235,0.325$, and $0.61 \mathrm{GHz}$ maps. The detection of radio emission in this area further indicates the presence of a magnetic field initially within the filament before the amplification due to merger event. The diffuse bridge emission could also be a part of the whole diffuse halo structure; however, more observations at frequencies below $0.325 \mathrm{GHz}$ with sensitivity below $0.69 \mathrm{mJy} /$ beam are needed with a radio facility like LOFAR in order to confirm this result and explain the mechanism that empowers relativistic electrons to emit on such large linear scales.

\subsection{Integrated radio spectra for cluster and discrete sources}

Radio spectra serve as important tools for understanding the origin of the relativistic electrons emitting nonthermal emission in galaxy clusters. The nonthermal synchrotron emission detected in cluster of galaxies follow a typical power law decay with $S(v) \propto v^{-\alpha}$, where $S(v)$ is the flux density, $v$ the frequency, and $\alpha$ the spectral index. To understand the connection between the halo and discrete field sources, it is important to understand their spectral nature. We analyzed the integrated radio spectra of the "Chair-shaped" filament, halo, and sources HT, B, C, C2, C4, and $\mathrm{F}$ by complementing the GMRT measurements at 0.235 and $0.614 \mathrm{GHz}$ with the flux densities available in the literature and archival data.

The integrated spectral index was computed using the relation

$$
\frac{\log S(v)}{\log (v)}=\alpha .
$$

A global spectral index of $\alpha_{4.86}^{0.235}=-1.33 \pm 0.01$ was obtained for the "Chair-shaped" filament by excluding source HT and $\mathrm{C} 4$, between 0.235 and $4.86 \mathrm{GHz}$, from high-resolution maps as shown in Fig. 3 and in agreement with Bonafede et al. (2009b). The error in the spectral index values is obtained from a linear fit to the data over the frequency range 0.235 to $4.86 \mathrm{GHz}$. Overall, the GMRT measurements agree both with the adjacent data points taken from the literature and the straight line fit, thus providing a consistent check of the flux density scale in our images.

The "Chair-shaped" filament shows a steep flux density spectrum between 0.235 and $4.86 \mathrm{GHz}$ (Fig. 3 top panel 1). Further analysis of spectral index variation between intermediate frequencies, $\alpha_{0.61}^{0.235}=-1.26 \pm 0.37$ and $\alpha_{4.86}^{1.4}=-1.35 \pm 0.1$ almost suggests flatness in the spectrum within error limits (Fig. 3 top panel 2). This flat response could be due to superposition of multiple blobs of relativistic particles emitting in radio. These blobs are likely to be formed in the regions with enhanced merger activity. The blobs gradually lose energy by adiabatic expansion, giving rise to a steep flux density spectrum $(\mathrm{En} \beta \operatorname{lin} \&$ Krishna 2001). In the case of a radio halo, a global spectral index, $\alpha_{4.86}^{0.235}=-0.98 \pm 0.03$, was computed from the lower resolution maps by excluding the sources F, C2, C1, and C3. Bonafede et al. (2009b) derived a spectral index, $\alpha_{4.86}^{1.42}=-1.27 \pm 0.02$, whereas van Weeren et al. (2009) derived a value $\alpha_{4.86}^{0.61}=-1.24 \pm 0.05$. Likewise van Weeren et al. (2009) computed a spectral in$\operatorname{dex}, \alpha_{1.42}^{0.61}=-1.04 \pm 0.13$, and we have derived a spectral in$\operatorname{dex}, \alpha_{0.61}^{0.235}=-1.17 \pm 0.37$, from our observations (Fig. 3 top panel). The error in the spectral index value between 0.235 and $0.61 \mathrm{GHz}$ was calculated using the noise in the image and flux density error measurements (Chandra et al. 2004). The analysis of spectral index suggests a steepness at higher frequencies due to spectral aging that might lead to high a frequency cut-off.
Furthermore, the steepness seen at lower frequencies could be due to more population of low-energy emitting relativistic particles available within the cluster volume (van Weeren et al. 2012).

The total flux of the radio halo at $0.61 \mathrm{GHz}$ and $0.235 \mathrm{GHz}$ are $\sim 162 \pm 0.23 \mathrm{mJy}$ and $\sim 492.5 \pm 0.52 \mathrm{mJy}$, respectively. As a result the monochromatic radio power at $1.42 \mathrm{GHz}$ (as similarly computed by van Weeren et al. 2012) using $\alpha_{0.61}^{0.235}=-1.17$ from our GMRT observations is, $P_{1.42}=9.88 \times 10^{25} \mathrm{~W} \mathrm{~Hz}^{-1}$. Thus, MACS $J 0717.5+3745$ is one of the most powerful radio halo, ever observed. The independent power estimate at $1.42 \mathrm{GHz}$ allows us to compare ours with the values available in the literature. The total power at $1.42 \mathrm{GHz}$ computed from our spectral index measurements is slightly less than in Bonafede et al. (2009b), since we have extrapolated the halo flux at $1.42 \mathrm{GHz}$ using $\alpha_{0.61}^{0.235}=-1.17$ and might have missed some additional diffuse emission due to limited sensitivity for a large angular scale. However, we did provid better flux and radio power estimates at $1.42 \mathrm{GHz}$ than do van Weeren et al. (2009) thanks to our high-sensitivity maps.

Important information about the magnetic field in this cluster can be derived from the spectral analysis performed above. The strength of the magnetic field can be estimated by assuming minimum energy densities in the radio sources (van Weeren et al. 2012). The minimum energy density (in units of $\mathrm{erg} / \mathrm{cm}^{3}$ ) is given by

$$
U_{\min }=\zeta\left(\alpha, v_{1}, v_{2}\right)(1+k)^{4 / 7} v_{0}^{-4 \alpha / 7}(1+z)^{(12-4 \alpha) / 7} I_{0} d^{-4 / 7}
$$

where $\zeta\left(\alpha, v_{1}, v_{2}\right)$, a constant tabulated in Govoni \& Feretti (2004), is $2.29 \times 10^{-13}, \alpha$ is $-1.17, I_{0}$ the surface brightness is $7.78 \mu \mathrm{Jy} \operatorname{arcsec}^{-2}$ at frequency $v_{0}=1420 \mathrm{MHz}, d$ the depth of the source is $1580(\mathrm{kpc})$, and $k$ the ratio of the energy in relativistic protons to that in electrons $=100$. A volume-filling factor of one has been assumed in the above equation. The equipartition magnetic field in terms of minimum energy is thus given as

$$
B_{\text {eq }}=\sqrt{\left(\frac{24 \pi}{7} U_{\min }\right)} .
$$

Using the derived quantities above, we compute the minimum energy, $U_{\min }=10.87 \times 10^{-13} \mathrm{erg} / \mathrm{s}^{3}$ and the equipartition magnetic field strength, $B_{\text {eq }}=3.43 \mu$ Gauss. The value of the magnetic field is slightly higher than in van Weeren et al. (2009) due to better flux and the linear size of the halo derived in our observations down to $0.235 \mathrm{GHz}$. This equipartition magnetic field strength is calculated on a fixed range of frequency $(10 \mathrm{MHz}-10 \mathrm{GHz})$; however, the upper and lower frequency limits depend on the energy of the radiating electrons (Brunetti et al. 1997; Beck \& Krause 2005). Considering the low and high energy cut-offs for the particle distribution and taking $\gamma_{\min } \leq \gamma_{\max }$ into account, the energy boundaries indicated by the Lorentz factor, the revised equipartition magnetic field strength $\left(B_{\text {eq }}^{\prime}\right)$ is

$$
B_{\mathrm{eq}}^{\prime} \sim 1.1 \gamma_{\min }^{\frac{1+2 \alpha}{3-\alpha}} B_{\mathrm{eq}}^{\frac{7}{2(3-\alpha)}}
$$

where $\gamma_{\min }$ is 100 . Thus the revised magnetic field strength, $B_{\mathrm{eq}}^{\prime}=6.49 \mu \mathrm{gauss}$, and is two times more than the standard equipartition estimate as expected (Govoni \& Feretti 2004).

The main goal of this work has been to constrain the spectral index of the halo in the MACS J0717.5 + 3745 cluster down to $0.235 \mathrm{GHz}$. The faint and extended nature of the halo makes it complicated to derive such a constraint on the spectral index distribution. Using the best comparison between sensitivity and 

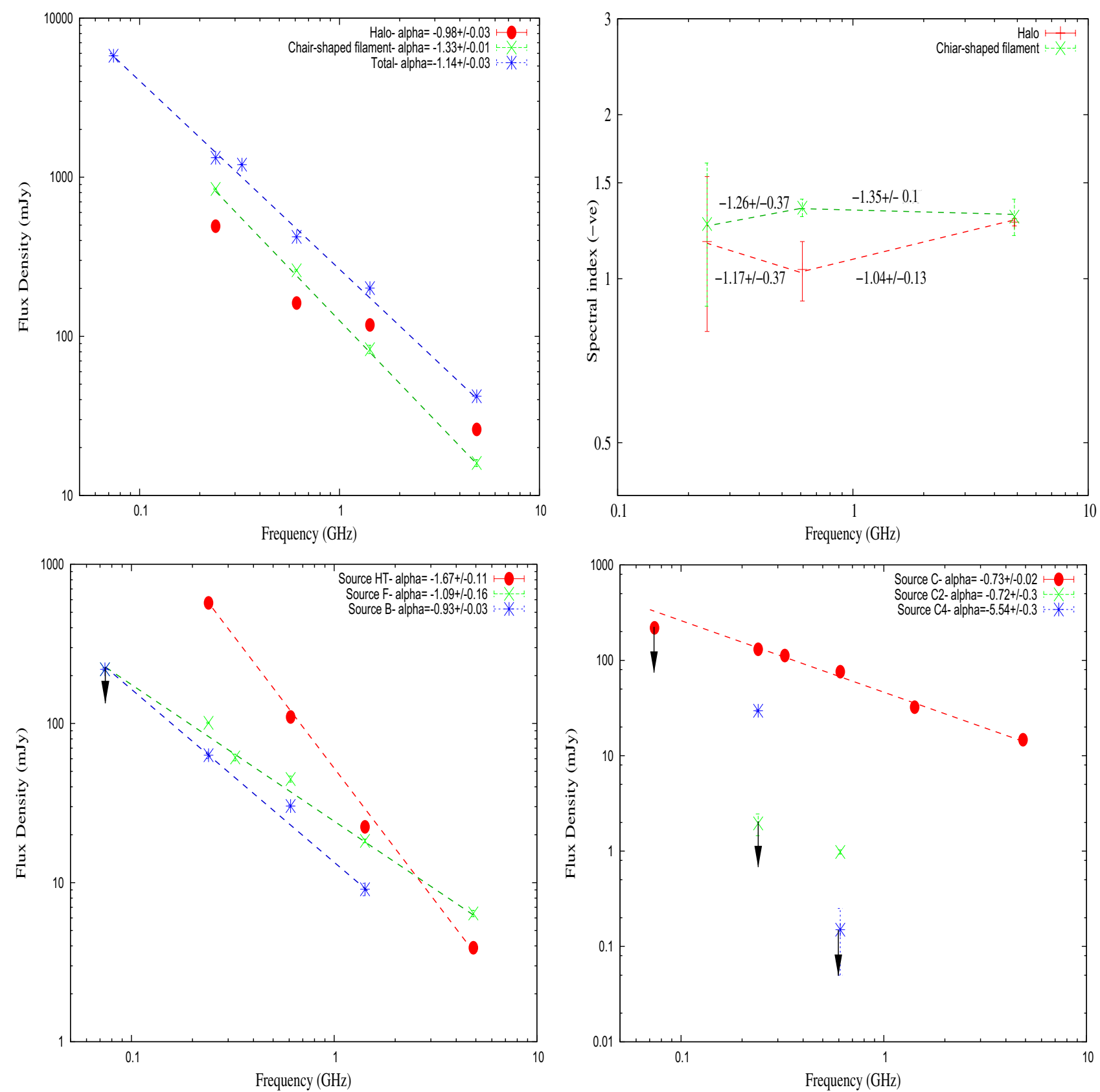

Fig. 3. Top left panel: integrated radio spectra of the halo, "Chair-shaped" filament source and total (halo+"Chair-shaped" filament source). Top right panel: spectral index variation with respect to frequency for the halo and "Chair-shaped" filament source. Bottom left panel: integrated radio spectra of extended sources HT, B and F. Bottom right panel: integrated radio spectra of compact source C, C2 and C4. The down arrows represent the $3 \sigma$ detection limit. Data from literature (Bonafede et al. 2009b; survey data) are plotted at 4.86, 1.4, 0.325 and $0.074 \mathrm{GHz}$ along with 0.61 and $0.235 \mathrm{GHz}$ GMRT data.

resolution in the 0.235 and $0.61 \mathrm{GHz}$ images, we have created the spectral index map for the halo at $13.46^{\prime \prime} \times 10.76^{\prime \prime}$. To take any effect due to their different sensitivity to the large angular structure into account, images at same UV-range were used to create similar resolution maps. In the Fig. 4 we present the spectral index image between 0.235 and $0.61 \mathrm{GHz}$ with $0.235 \mathrm{GHz}$ radio contours overlaid at a resolution of $13.3^{\prime \prime} \times 10.7^{\prime \prime}$. The uncertainties in the computed spectral index map are also shown in Fig. 5 and range from 0.09 to 0.9 . The error in the spectral index estimate between 0.61 and $0.235 \mathrm{GHz}$, as discussed earlier is consistent with the spectral index error map. The spectral index is only shown in the regions where the flux is greater than $3 \sigma$ at both frequencies. Additional errors in the spectral index could be noticed owing to different calibration errors and UV-coverage at both the frequencies. The overall radio spectrum of the halo is steep. The spectral index ranges from $\alpha \sim-0.85$, close to the cluster center, up to about $\alpha \sim-2.3$ at the edge. The analysis of the spectral index profile in the "Chair-shaped" filament structure shows a variation from -0.92 up to -1.5 . The flatter spectral index in this filament is coincident with the high temperatures in merging regions (Ma et al. 2008), and this agrees with the ICM temperature and flatness in the spectra correlation derived via Govoni et al. (2004). Source HT shows variation in spectral index from -0.95 near the head region to -1.8 towards the tail region. Such a spectral steepening from the head towards the lobes has been seen in radio galaxies and is believed to be due 


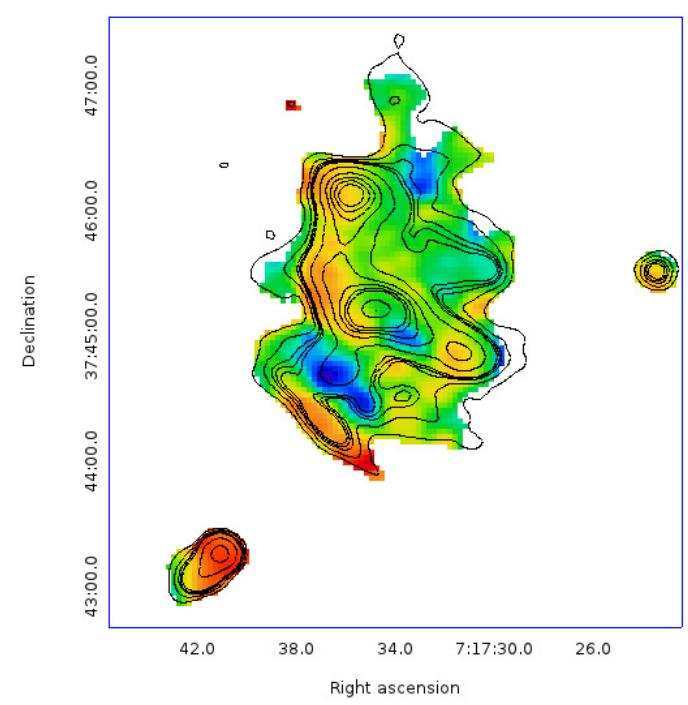

Fig. 4. Spectral index map between 0.235 and $0.61 \mathrm{GHz}$ at a resolution of 13.3" $\times 10.7^{\prime \prime}$. Contours are from the $0.61 \mathrm{GHz}$ image and drawn at a level of $[1,2,3,4,16,32, \ldots] \times 0.15 \mathrm{mJy}^{\text {beam }}{ }^{-1}$.
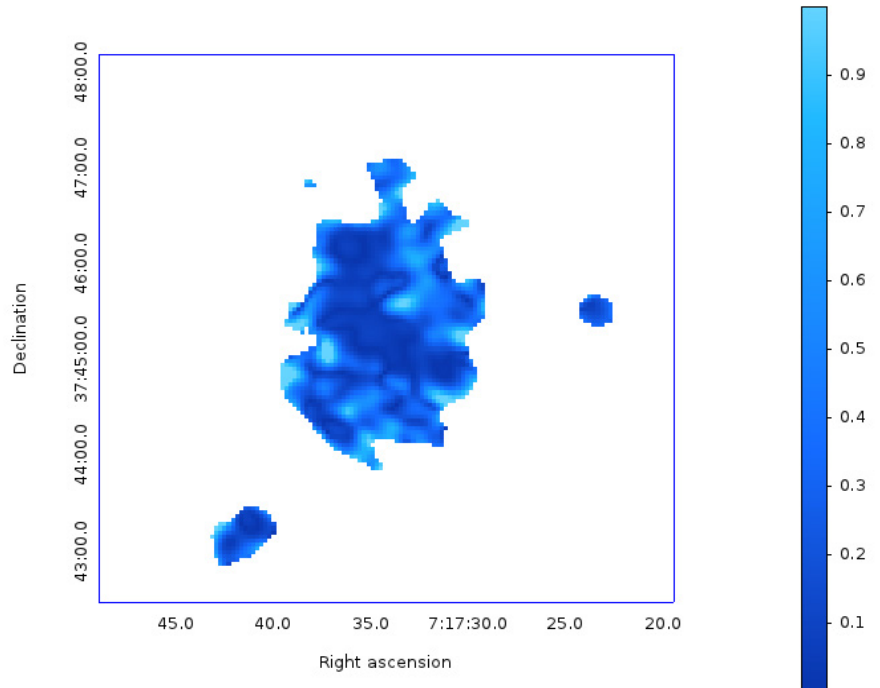

Fig. 5. Spectral index error map between 0.235 and $0.61 \mathrm{GHz}$ image. The error map shows the uncertainties in the spectral index based on the rms noise in both the radio images.

to spectral aging due to synchrotron and inverse Compton (IC) losses. As source HT has much steeper spectra than the "Chairshaped" filament, the radio emission from this region is expected to have a different origin.

The extended sources $\mathrm{F}, \mathrm{B}$, and $\mathrm{C}$ present a power law radio spectrum with a spectral index of about $\alpha=-1.09 \pm 0.16$, $\alpha=-0.93 \pm 0.03$ and $\alpha=-0.73 \pm 0.02$ (Fig. 3 bottom panel). This is the usual value for active radio galaxies. The study of the spectral index suggests, that radio emission in these galaxies are less steep than in the halo and the "Chair-shaped" filament structure, so it is produced by different populations of relativistic electrons.

\section{Discussion}

Most galaxy clusters that host radio halos and bright filaments also show signatures of recent or ongoing merger activities (Ferrari et al. 2008). The massive cluster MACS J0717.5+3745

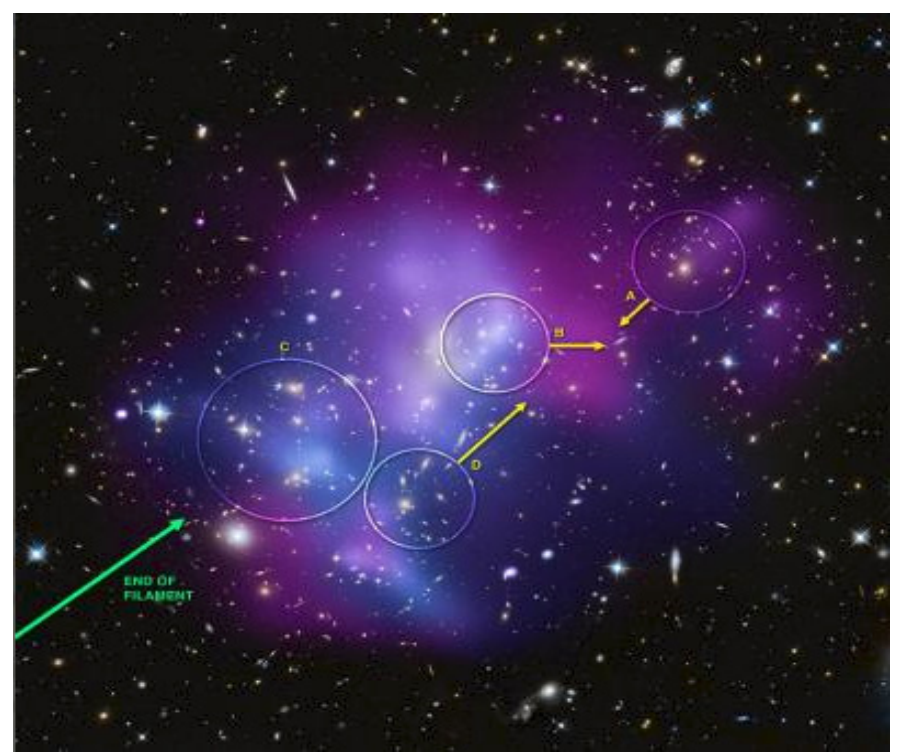

Fig. 6. MACS J0717.5 + 3745: Chandra color image on HST showing galaxies in 4 different clusters involved in collision, with direction of motion for the three fastest moving clusters. The length of the arrow shows the approximate speed in a direction perpendicular to the line of sight. The direction of motion of the cluster appears roughly parallel to the direction of the filament (Credit: X-ray, NASA/CXC/IfA/C. Ma et al. 2008, 2009; Optical, NASA/STScI/IfA/C. Ma et al. 2008, 2009). The color represents the temperature scale of the cluster, where the blue color indicates the hottest region and reddish purple color indicates the cooler region and the temperatures in between are represented by purple.

is a complex case involving a triple merger (Ma et al. 2008, 2009). The implications for the spectral index trends in the diffuse radio emission in MACS $J 0717.5+3745$ from the proposed origins in merger shocks is further explored here.

\subsection{Cluster dynamics and ICM temperature}

A schematic representation of the Chandra map of MACS $J 0717.5+3745$ (available from NASA/CXC/IfA/C, Ma et al. 2008, 2009) is shown by the color scale over HST map in (Fig. 6), where blue indicates the hottest region $(T=24.3 \pm$ $5.3 \mathrm{keV})$, reddish purple indicates the cooler region $(T=7.3 \pm$ $0.7 \mathrm{keV})$, and the temperatures in between are represented by purple $(T=16.1 \pm 4.0 \mathrm{keV})(\mathrm{Ma}$ et al. 2008, 2009). Figure 7 shows the radio contours overlaid on the Chandra color image. We have also identified four different regions of interest based on optical data, for further analysis within the halo marked as CL1, CL2, CL3, and CL4 shown in Fig. 7. The optical and X-ray studies (Ma et al. 2008, 2009) suggest that the cluster region marked as CL1 $(T \sim 8.7 \pm 1.2 \mathrm{keV})$ has already passed through the main cluster previously in the opposite direction and is likely falling back, whereas the cluster CL2 $(T \sim 8.2 \pm 1.5 \mathrm{keV})$ has unclear origin and has higher speed along the line of sight than do the other clusters. The cluster region labeled as CL3 $(T \sim 24.3 \pm 5.3 \mathrm{keV})$ is identified as the core of the main cluster based on the good alignment between the galaxies and hot gas for cluster CL3, along with its motion compared to MACS $J 0717.5+3745$ as a whole. While region CL4 $(T \sim 5.7 \pm 0.8 \mathrm{keV})$ is a cooler (redder) region of gas and has likely survived from before the collision in the cluster. CL1 and CL4 are also suggested to originate in the filament. The large region of relatively hot gas (shown in blue) is seen to extend from 


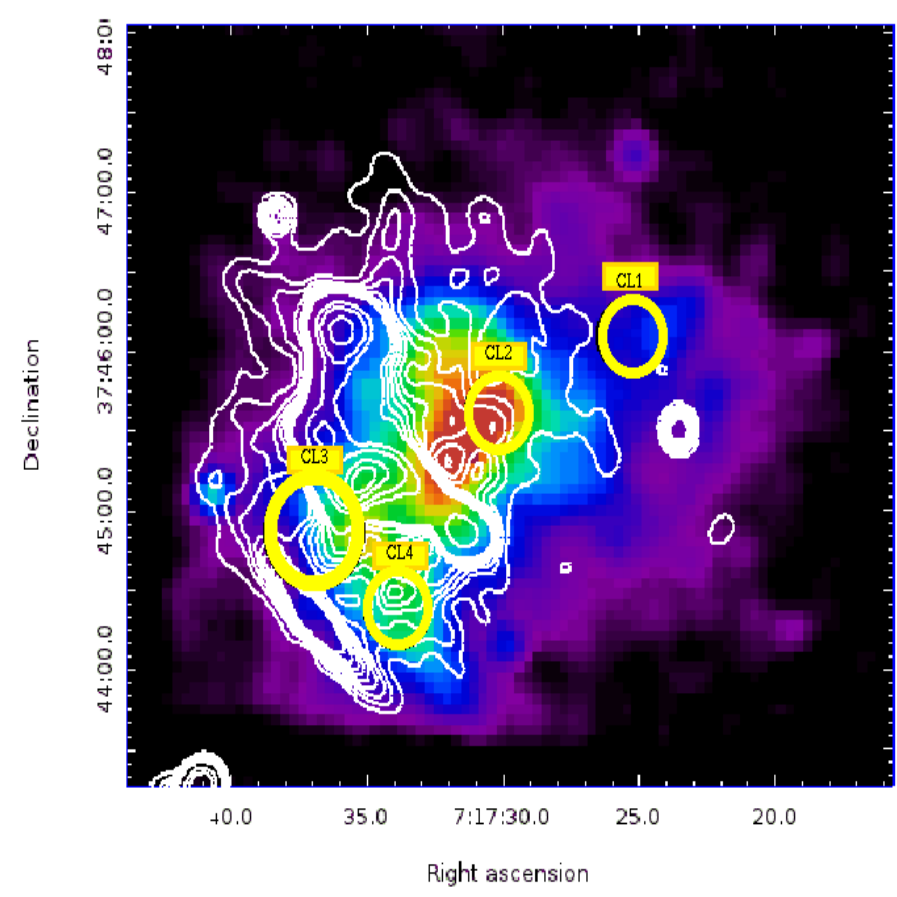

Fig. 7. MACS $J 0717.5+3745$ : GMRT contours at $0.61 \mathrm{GHz}$ overlaid on X-ray Chandra map with 4 different cluster regions involved in collision indicated by circles similar to Fig. 6 .

the left side of cluster CL3 to the right side of region CL4 and may be caused by heating as significant quantities of gas from the filament plough into the main cluster. Thus the cooler region is detected towards the outer edge, while the hotter region is detected at the center where merging events occur. Other clusters CL2 and CL4 are identified as the cool cores of clusters merging with the main body of MACS J0717.5 + 3745 (Ma et al. 2008, 2009).

A spatial comparison of the low-frequency radio image and the X-ray brightness image is shown in Fig. 7. The "Chairshaped" filament structure with flat spectral index as seen in Sect. 3.4 (Fig. 3 top right) is well placed between the merging region of the four clusters where high ICM temperature are detected $(24.3 \pm 5.3 \mathrm{keV}$, Ma et al. 2009). The merging events cause heating of the thermal plasma due to shock, which in turn reaccelerates the relativistic particles and amplifies the magnetic field (En $\beta$ lin \& Krishna 2001). The radio halo with a steep spectral index of $\alpha_{0.235}^{0.61}=-1.17 \pm 0.37$ (Fig. 3 top right), from the central region near the "Chair-shaped" filament structure towards the outer edge, traces the overall outer edge and cooler region of the cluster. A correlation between the radio-halo, integrated spectral index and the average X-ray gas temperature for a large sample of nearby radio halos has been derived recently by Giovannini et al. (2009). Hot clusters $(T>10 \mathrm{keV})$ show an average spectral index $=-1.1$ to -1.2 , and cold clusters $(T \leq 8 \mathrm{keV})$ show steep spectra (average spectral index $=-1.7)$, according to the correlation derived by Giovannini et al. (2009) and Venturi et al. (2013). The radio spectrum of present radio halo with $\alpha \sim-1.17$ derived from our low-frequency observations further confirms the result that MACS J0717.5+3745 is a high-temperature merging cluster, $T=10.2 \pm 2.4 \mathrm{keV}(\mathrm{Ma}$ et al. 2009) that shows agreement with the correlation between the radio-halo, integrated spectral index and the average $\mathrm{X}$-ray gas temperature.

Based on the mass of the cluster Venturi et al. (2013) found that the radio halos show different spectra depending on

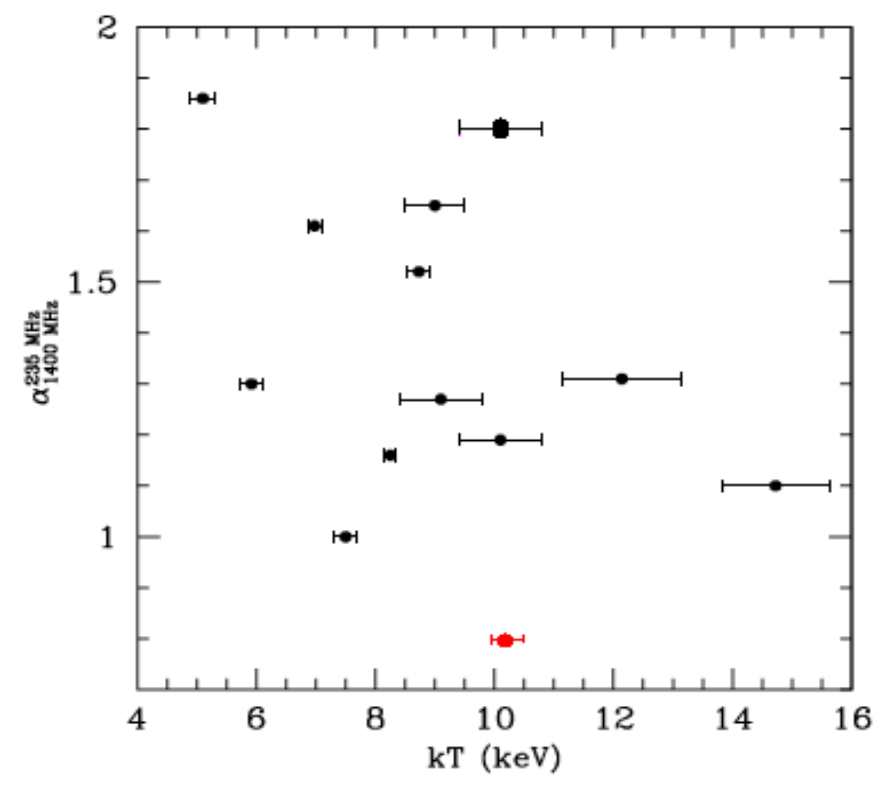

Fig. 8. Spectral index of radio halos in the $235 \mathrm{MHz}-1.4 \mathrm{GHz}$ interval as function of the cluster X-ray temperature (adapted from Venturi et al. 2013 and Giacintucci et al., in prep.).The red points mark the position of MACS J0717.5 + 3745.

the energy release into particle re-acceleration during mergers. The trend toward flatter spectra is seen in massive hot clusters $(T>8 \mathrm{keV})$ with halos, while moderately massive clusters ( $T \sim 5-8 \mathrm{keV})$ shows halos with both steep and flat spectra (Cassano et al. 2006; Cassano et al. 2010). Figure 8 reports the distribution of spectral index $\left(\alpha_{1400 \mathrm{MHz}}^{235}\right)$ in radio-halo clusters versus the X-ray temperature, $\mathrm{kT}(\mathrm{keV})$ adapted from Venturi et al. (2013). The position of MACS J0717.5 + 3745 is shown by a red point with $k T=10.2 \pm 2.4 \mathrm{keV}$. Thus, it is the most distant massive cluster with radio halo showing spectral index, $\alpha_{1.42}^{0.235}=-0.8$, less steep than other hotter clusters, suggesting its complex nature. Because it is less steep than the massive galaxy clusters, MACS J0717.5+3745, is an interesting candidate that provides an upper limit for our understanding of mass-spectral index correlation of giant radio halos in distant massive hotter clusters with multiple mergers.

\subsection{Spectral index distribution within the halo}

We then carried out spectral index analysis of specific regions CL2, CL3, and CL4 where halo emission is detected at radio wavelengths and three merging clusters were detected from optical observations (Limousin et al. 2012). Figure 9 shows Chandra contours overlaid on a radio spectral index map between 0.61 and $0.235 \mathrm{GHz}$. A variation in the spectral index distribution is clearly seen to be coincident with the dynamical state of the cluster derived from X-ray images. The spectral index $\left(\alpha_{0.235}^{0.61}\right)$ map analysis for Region CL2 shows a steepness variation of -1.65 towards the outer edge and gradually decreases to -1.3 towards the "Chair-shaped" filament. In the case of region CL3, the steepness in spectral index varies from -2.3 from the outer edge to the less steep value of -1.2 towards the "Chair-shaped" filament. For region CL4, the spectral steepness is -0.98 towards the "Chair-shaped" filament and increases to -1.3 towards the outer edge. Thus, from the overall spectral index distribution analysis, we found that the spectral steepening gradually increases from the central region near the "Chair-shaped" filament towards the outer edge. Thus outer steep spectral index region is 


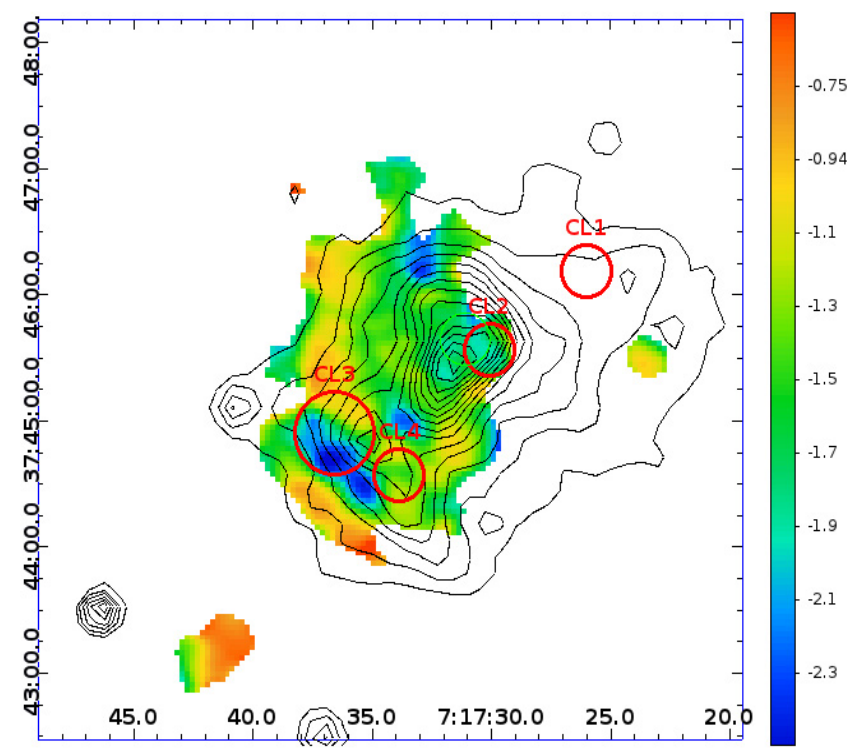

Fig. 9. MACS $J 0717.5+3745$ : GMRT spectral index map between 0.61 and $0.235 \mathrm{GHz}$ with overlaid X-ray contours from Chandra.

co-spatial with the colder region within MACS J0717.5 + 3745 and confirms Ma et al. (2009). The hotter central region is also coincident with the merger axis of the cluster, so that less steepening is seen towards the "Chair-shaped" filament structure. Furthermore, on comparison with Fig. 8 and discussion in Sect. 4.1, it is seen that region CL3, lying near the merger axis of the cluster, shows the highest temperature and a broader range of spectral index variation owing to the shockwaves encountered during the merger event. The colder regions CL2 and CL4 located far from the active site, towards the outer edge of the cluster, show a narrow range of spectral index variation.

In addition to the above spectral steepening trend towards the outer edge, the diffuse radio emission in the halo also shows less spectral steepness at lower frequencies (Fig. 3 top panel) with $\alpha_{0.235}^{0.61}=-1.17 \pm 0.37$ as compared to higher frequencies $\alpha_{4.86}^{0.61}=-1.24 \pm 0.05$ (van Weeren et al. 2009), though this comparison cannot lead to firm conclusions due to big error bars the spectral index values are actually consistent within error limits. Such an upturn in the spectrum of the halo at lower frequencies has been measured in A2256 (Kale \& Dwarakanath 2010; van Weeren et al. 2012). This trend towards less steepening in the lower frequency spectrum could be due to superposition of at least two or more spectra having a different steepness that adds up to flatter spectra at low frequencies. In the case of MACS $J 0717.5+3745$, the triple merger events could be the three epochs at which the electrons were accelerated with different input energy giving rise to varied spectral steepness. However, detailed simulations are required to establish the exact mechanism of acceleration of electrons to relativistic energies and the chronology between the three merger events. Typically the synchrotron spectrum is expected to steepen at higher frequencies due to energy losses. Thus putting together the spectral information across the frequency range of 0.235 to $4.86 \mathrm{GHz}$ for the halo region, a scenario consistent with a high-frequency steepening due to the energy losses and a low-frequency less steepening due to a secondary electron population, emerges as seen in A2256 (Kale \& Dwarakanath 2010; van Weeren et al. 2012).

An alternative scenario could be that the emission of the "Chair-shaped" filament could be projected on the halo emission, causing a flatter spectrum at lower frequencies. The complex spectrum may simply result from a superposition of two components ("Chair-shaped" filament + halo). Furthermore the steeper spectral indices in the $1.42-4.86 \mathrm{GHz}$ integrated spectra could be the act of the synchrotron spectrum aging due to energy losses. New observations with LOFAR at lower frequencies $(<0.235 \mathrm{GHz})$ and complementary observations at high frequencies are needed to determine the shape of the spectrum of the halo (cut-off), to better constrain its origin and image the entire extent of the diffuse bridge emission in the cluster at higher sensitivity.

\section{Conclusion}

In this work we present a study of the radio emission in MACS J0717.5 + 3745 with more sensitive observations at $0.61 \mathrm{GHz}$ and a new a map $0.235 \mathrm{GHz}$ with the GMRT. We have confirmed the detection of the radio halo within the massive galaxy cluster down to $0.235 \mathrm{GHz}$. It is the most powerful and distant cluster ever observed. The results achieved from these observation are summarized below.

- A bright "Chair-shaped" filament structure is detected at $0.235 \mathrm{GHz}$ on a linear scale of $853 \mathrm{kpc}$ and at $0.61 \mathrm{GHz}$ at $772 \mathrm{kpc}$. This filament is enclosed in a diffuse halo structure of $1.6 \mathrm{kpc}$ at $0.235 \mathrm{GHz}$ and $1.5 \mathrm{kpc}$ at $0.61 \mathrm{GHz}$. The complex morphology of the radio cluster indicates the ongoing merger activity within the cluster, supported by optical and X-ray studies.

- A radio power of $0.98 \times 10^{26} \mathrm{WHz}^{-1}$ at $1.4 \mathrm{GHz}$ is estimated from the steep radio spectral index of -1.17 derived from GMRT observations with a hint of steepening at lower frequencies.

- Using standard equipartition magnetic field assumption, we derived an equipartition magnetic field strength of $3.43 \mu \mathrm{G}$ for the radio halo. A somewhat higher value with a revised magnetic field strength of $6.49 \mu \mathrm{G}$ is obtained by using lower and higher energy cutoffs instead of fixed frequency cutoffs.

- We also confirm the presence of additional diffuse bridge emission of $\sim 3.7 \mathrm{Mpc}$ connecting the central radio halo with the discrete field sources $\mathrm{B}$ and $\mathrm{C}$, indicating there is a magnetized ICM at this redshift.

- The location of the bright "Chair-shaped" filament coincides with regions in the cluster having higher temperatures. The major axis of this filament is located perpendicular to the merger axis of the system. This shows that the "Chairshaped" filament is probably the result of a large-scale shock wave within the cluster where are particles are accelerated during merger events.

- In addition to the halo spectrum, we also analyzed the properties of the cluster discrete sources, specifically sources HT, B, C, C2, C4, and F. Sources HT and B are head-tail sources, while sources C, C2, C4 are unresolved and source $\mathrm{F}$ is extended with core and lobe morphology. The spectral index analysis, along with NED counterparts, suggests that these are radio galaxies with different origins of radio emission as compared to the radio halo and bright central filament within the cluster.

Acknowledgements. We would like to thank the anonymous referee for the careful review of the paper that helped us to improve the clarity of our results. We thank the staff of the GMRT, who made these observations possible. The GMRT is run by the National Centre for Radio Astrophysics of the Tata Institute of Fundamental Research. This research had made extensive use of the NASA/IPAC extragalactic sources data base NED, Data from the NASA/NVSS survey, and the public archives from the HST and Chandra. Chiara Ferrari acknowledges financial support by the Agence Nationale de la Recherche through grant ANR-09-JCJC-0001-01. 


\section{References}

Bagchi, M., Feretti, L., \& Giovannini, G. 2003, A\&A, 400, 465

Beck, R., \& Krause, M. 2005, Astron. Nachr., 326, 414

Becker, R. H., White, R. L., Helfand, D. J., Greeg, M. D., et al. 1994, A\&AS, 185

Becker, R. H., White, R. L., \& Helfand, D. J. 1995, ApJ, 450, 559

Bonafede, A., Feretti, L., Giovannini, G., et al. 2009a, A\&A, 503, 707

Bonafede, A., Giovannini, G., Feretti, L., Govoni, F., \& Murgia, M. 2009b, A\&A, 494, 429

Boselli, A., \& Gavazzi, G. 2006, PASP, 118, 517

Briggs, D. S. 1995, Ph.D. Thesis, New Mexico Institute of Mining Technology, Socorro, New Mexico, USA

Brunetti, G., Setti, G., \& Comastri, A. 1997, A\&A, 325, 898

Brunetti, G., Setti, G., Feretti, L., \& Giovannini, G. 2001, MNRAS, 320, 365

Brunetti, G., Giacintucci, S., Cassano, R., et al. 2008, Nature, 455, 944

Bruggen, M., van Weeren, R. J., \& Rottgering, H. J. A. 2011, Mem. Soc. Astron. It., 82, 627

Cassano, R., Brunetti, G., \& Setti, G. 2006, MNRAS, 369, 1577

Cassano, R., Ettori, S., Giacintucci, S., et al. 2010, ApJ, 721, L82

Chandra, P., Ray, A., \& Bhatnagar, S. 2004, ApJ, 612, 974

Cohen, A. S., Lane, W. M., Cotton, W. D., et al. 2007, AJ, 134, 1245

Condon, J. J., Cotton, W. D., Greisen, E. W., et al. 1998, AJ, 115, 1693

Cornwell, T. J., \& Perley, R. A. 1992, A\&A, 261, 353

Drury, L. O. 1983, Rep. Prog. Phys., 46, 973

Dursi, L. J., \& Pfrommer, C. 2008, ApJ, 677, 993

Ebeling, H., Edge, A. C., \& Henry, J. P. 2001, ApJ, 553, 668

Ebeling, H., Barrett, E., \& Donovan, D. 2004, ApJ, 609, L49

Ebeling, H., Barrett, E., Donovan, D., et al. 2007, ApJ, 661, L33

Edge, A. C., Ebeling, H., Bremer, M., et al. 2003, MNRAS, 339, 913

Ensslin, T. A., Biermann, P. L., Klein, U., \& Kohle, S. 1998, A\&A, 332, 395

En/lin, T. A., \& Gopal-Krishna 2001, A\&A, 366, 26

Fanaroff, B. L., \& Riley, J. M. 1974, MNRAS, 167, 31

Feretti, L. 2002, in Universe Low Radio Frequency. IAU Symp., 199, 133

Feretti, L. 2005, Adv. Space Res., 36, 729
Feretti, L., \& Giovannini, G. 2008, Lect. Notes Phys. 740, eds. M. Plionis, O. L'opez-Cruz, \& D. Hughes, (Dordrecht: Springer), 143

Feretti, L., Giovannini, G., Govoni, F., \& Murgia, M. 2012, A\&ARv, 20, 54

Ferrari, C., Govoni, F., Schindler, S., Bykov, A. M., \& Rephaeli, Y. 2008, SSRv, 134,93

Finoguenov, A., Sarazin, C. L., Nakazawa, K., et al. 2010, ApJ, 715, 1143

Giovannini, G., \& Feretti, L. 2004, J. Korean Astron. Soc., 37, 323

Giovannini, G., Bonafede, A., Feretti, L., et al. 2009, A\&A, 507, 1257

Govoni, F., \& Feretti, L. 2004, IJMPD, 13, 1549

Govoni, F., Murgia, M., Feretti, L., et al. 2005, A\&A, 430, L5

Govoni, F., Murgia, M., Markevitch, M., et al. 2009, A\&A, 499, 371

Jaffe, W. J., \& Perola, G. C. 1973, A\&A, 26, 423

Kale, R., \& Dwarakanath, K. S. 2009, ApJ, 699, 1883

Kale, R., \& Dwarakanath, K. S. 2010, ApJ, 718, 939

Limousin, M., Ebeling, H., Richard, J., et al. 2012, A\&A, 544, A71

Ma, C.-J., Ebeling, H., Donovan, D., \& Barrett, E. 2008, ApJ, 684, 160

Ma, C.-J., Ebeling, H., \& Barrett, E. 2009, ApJ, 693, L56

Markevitch, M., \& Vikhlinin, A. 2007, Phys. Rep., 443, 1

Markevitch, M., Gonzalez, A. H., David, L., et al. 2002, ApJ, 567, L27

Miniati, F., Jones, T. W., Kang, H., \& Ryu, D. 2001, ApJ, 562, 233

Mroczkowski, T., Dicker, S., Sayers, J., et al. 2012, ApJ, 761, 47

Murgia, M., Eckert, D., Govoni, F., et al. 2010, A\&A, 514, A76

Parrish, I. J., Quataert, E., \& Sharma, P. 2009, ApJ, 703, 96

Perley, R. A. 1989, in Synthesis Imaging in Radio Astronomy, eds. R. A. Perley,

F. R. Schwab, \& A. H. Bridle, ASP Conf. Ser., 6, 259

Petrosian, V. 2001, ApJ, 557, 560

Rengelink, R. B., Tang, Y., de Bruyn, A. G., et al. 1997, A\&AS, 124, 259

van Weeren, R., Rottgering, H. J. A., Bruggen, M., \& Cohen, A. 2009, A\&A, 505,991

van Weeren, R., Rottgering, H. J. A., Bruggen, M., et al. 2010, Science, 330, 347

van Weeren, R., Rottgering, H. J. A., Rafferty, D. A., Pizzo, R., \& Bonafede, A. 2012, A\&A, 543, A43

Venturi, T., Giacintucci, S., Brunetti, G., et al. 2007, A\&A, 463, 937

Venturi, T., Giacintucci, S., Dallacasa, D., et al. 2008, A\&A, 484, 327

Venturi, T., Giacintucci, S., Dallacasa, D., et al. 2013, A\&A, 551, A24

Zitrin, A., Broadhurst, T., Rephaeli, Y., Sadeh, S. 2009, ApJ, 707, L102 